\title{
HOMOTOPY GROUPS OF THE ISOTROPY GROUPS OF ANNULUS
}

\author{
JONG P. LEE
}

\begin{abstract}
We compute the isotopy groups of various subspaces of the isotropy group at an interior point of an annulus. We also prove that if $a$ and $x$ are interior points of a disk $D$, then $\pi_{0}[H(D-a, x)]=Z_{2}$ and $\pi_{n}[H(D-a, x)]=0$ for $n \geqq 1$ where $H(D-a, x)$ is the isotropy group at $x$.
\end{abstract}

1. Introduction. Let $X$ be a topological space, and let $H(X)$ denote the group of homeomorphisms of $X$ onto itself topologized by the compact open topology. The isotropy group at $x \in X$ will be denoted by $H(X, x)=\{h \in H(X) \mid h(x)=x\}$. The arc-component of the identity $H_{0}(X)$ is a normal subgroup of $H(X)$ and $H(X) / H_{0}(X)=\pi_{0}[H(X)]$ is the group of the arc-components of $H(X)$, which is called the isotopy group of $H(X)$. The isotopy groups for the subspaces of $H(X)$ are similarly defined. In this note we compute the isotopy groups of various subspaces of the isotropy group at an interior point of an annulus, and $\pi_{n}[H(D-a, x)]$ for $n \geqq 0$ where $D$ is a disk and $a, x \in \operatorname{Int}(D)$.

2. Preliminaries. We state some fundamental lemmas which will be needed in the sequel.

LEMMA 2.1. The space of homeomorphisms of a closed n-cell onto itself which leave the boundary of the n-cell pointwise fixed is contractible [1, p. 406].

LEMMA 2.2. The space of homeomorphisms of an annulus onto itself, which leave one of the boundary curves pointwise fixed, is contractible $[4, p .526]$.

LemmA 2.3. If $A$ is an annulus, then $\pi_{0}[H(A)]=Z_{2} \times Z_{2}[6, p$. 924].

Definition 2.4. An isotopy between imbeddings $f_{0}$ and $f_{1}$, defined on a space $X$ into a space $Y$, is a continuous map $G: X \times I \rightarrow Y$ such that the function $G_{t}$ defined by $G_{t}(x)=G(x, t)$ is a homeomorphism for each

Received by the editors June 28, 1972 and, in revised form, April 7, 1973.

AMS (MOS) subject classifications (1970). Primary 57E05; Secondary 55E05.

Key words and phrases. Homeomorphism, homotopy group, isotopy group, isotropy group, winding number, homeotopy exact sequence.

(c) American Mathematical Society 1974 
$t \in I=[0,1]$, and for all $x \in X, G(x, 0)=f_{0}(x)$ and $G(x, 1)=f_{1}(x)$. If each $G_{t}$ is also surjective and $G_{0}$ is the identity, then $G$ is an ambient isotopy. An isotopy which moves no point on $\mathrm{Bd}(X)$ is called a $B$-isotopy. $h \approx g\left[h \approx_{B} g\right.$ ] will denote that $h$ is isotopic [B-isotopic] to $g$.

LEMma 2.5. Let $f_{0}, f_{1}$ be imbeddings of $S^{1}$ in $\operatorname{Int}(X)$ such that $f_{0} \simeq f_{1}$ : $S^{1}, * \rightarrow X, *$, where $X$ is a 2-manifold. If $f_{0}\left(S^{1}\right)$ does not bound a disk or Möbius band in $X$, then there is an ambient isotopy between $f_{0}$ and $f_{1}$ keeping the base point fixed and which is fixed outside a compact subset of $\operatorname{Int}(X)[2, p .91]$.

Let $A=S^{1} \times I$ and $H^{2}(A)=\{h \in H(A) \mid h=e$ on $\operatorname{Bd}(A)\}$. H. Gluck [3] defined the winding number for a homeomorphism $h \in H^{2}(A)$ as follows. Let $\eta$ be the isomorphism of $\pi_{1}\left(S^{1}, 0\right)$ with $Z$ which takes the class of the path $f(t)=t$ onto 1 . Let $\alpha$ be any path in $S^{1} \times I$ from $(0,0)$ to $(0,1)$ and $P_{1}: S^{1} \times I \rightarrow S^{1}$ the natural projection. Then $P_{1}(\alpha)$ is a closed path in $S^{1}$ based at 0 . Hence $\left[P_{1}(\alpha)\right]$ is an element of $\pi_{1}\left(S^{1}, 0\right)$ and $\eta\left(\left[P_{1}(\alpha)\right]\right)=\omega(\alpha)$ is an integer. The integer $\omega(h \alpha)-\omega(\alpha)$ is independent of the path $\alpha$ for any $h \in H^{2}(A)$.

DEFINITION 2.6. Let $h$ be a homeomorphism in $H^{2}(A)$ and $\alpha$ a path in $A$ from $(0,0)$ to $(0,1)$. Then the integer $W[h ; A]=\omega(h \alpha)-\omega(\alpha)$ is called the winding number of $h$ on $A$.

We note that $W$ defines a homomorphism $W: H^{2}(A) \rightarrow Z$. But it is shown that the kernel of $W$ is the arc-component of the identity $H_{0}^{2}(A)$ and thus $W$ is in fact an isomorphism of $H^{2}(A)$ onto $Z$ [3, p. 314].

The space $H(X)$ of a manifold $X$ is a fiber bundle over $\operatorname{Int}(X)$ with fiber $H(X, x)$. The homotopy sequence of this bundle is exact and McCarty [5] obtained the following exact sequence which is called the homeotopy exact sequence of $X$,

$\cdots \stackrel{I_{*}^{\prime}}{\longrightarrow} \pi_{n+1}(X, x) \stackrel{d_{*}}{\longrightarrow} \pi_{n}[H(X, x)] \stackrel{i_{*}}{\longrightarrow} \pi_{n}[H(X)] \stackrel{P_{*}}{\longrightarrow} \pi_{n}(X, x) \longrightarrow \cdots$

$\cdots \stackrel{P_{*}}{\longrightarrow} \pi_{1}(X, x) \stackrel{d_{*}}{\longrightarrow} \pi_{0}[H(X, x)] \stackrel{i_{*}}{\longrightarrow} \pi_{0}[H(X)] \stackrel{P_{*}}{\longrightarrow} 0$,

where, if $X$ is locally compact, locally connected and Hausdorff, $P_{*}\left(\pi_{1}[H(X)]\right)$ is the center of $\pi_{1}(X, x)$ [5, p. 302].

3. Isotopy groups. In what follows $A$ will denote an annulus which we take as the cylinder $S^{1} \times I$ with the notations $C_{1}=S^{1} \times 0$ and $C_{2}=S^{1} \times 1$, $A_{1}=S^{1} \times\left[0, \frac{1}{2}\right]$ and $A_{2}=S^{1} \times\left[\frac{1}{2}, 1\right]$.

THEOREM 3.1. Let $a$ be an interior point of $A$. Then

(i) $\pi_{0}[H(A, a)]=Z_{2} \times Z_{2}$,

(ii) $\pi_{0}\left[H^{1}(A, a)\right]=Z$, where $H^{1}(A, a)=\left\{h \in H(A, a) \mid h=e\right.$ on $\left.C_{1}\right\}$,

(iii) $\pi_{0}\left[H^{2}(A, a)\right]=Z \times Z$, where $H^{2}(A, a)=\left\{h \in H(A, a) \mid h=\right.$ e on $\left.C_{1} \cup C_{2}\right\}$. 
Proof. (i) In the homeotopy exact sequence of $A, P_{*}: \pi_{1}[H(A)] \rightarrow$ $\pi_{1}(A, a)$ is onto. Thus the sequence yields $\pi_{0}[H(A, a)]=\pi_{0}[H(A)]$ and (i) holds by Lemma 2.3 .

(ii) Let $a=\left(0, \frac{1}{2}\right)$ and $\gamma$ be the closed $\operatorname{arc} S^{1} \times \frac{1}{2}$ in $A$. We note that every $h \in H^{1}(A, a)$ is orientation preserving on $A$ since it is the identity on the boundary curve $C_{1}$. Thus $h(\gamma)$ does not bound a disk or Möbius band and $h(\gamma) \simeq \gamma$ fixing the point $a$, since the homotopy group $\pi_{1}(A, a)=Z$ has only the identity and inverse automorphisms and $h$ must induce the identity automorphism of $\pi_{1}(A, a)$. Lemma 2.5 implies that there is an ambient isotopy $G_{t}: A, a \rightarrow A, a(0 \leqq t \leqq 1)$ such that $G_{0}=e$ and $G_{1}=h$ on $\gamma$. Since $G_{1}^{-1} h=e$ on $\gamma$, Lemma 2.2 implies that $G_{1}^{-1} h \mid A_{2} \approx e$ on $A_{2}$ by an isotopy which is the identity on the closed arc $\gamma$ and moves only on the boundary curve $C_{2}$.

In $A_{1}$, since $G_{1}^{-1} h=e$ on $\gamma \cup C_{1}$, the isotopy classes of the restricted homeomorphisms $\left\{G_{1}^{-1} h \mid A_{1}\right\}$, for all $h \in H^{1}(A, a)$ and the above defined homeomorphisms $G_{1}$, are $Z$ classified by the winding numbers $\left\{W\left[G_{1}^{-1} h \mid A_{1} ; A_{1}\right]\right\}$. But a homeomorphism $G_{1}^{-1} h$ such that $W\left[G_{1}^{-1} h \mid A_{1} ; A_{1}\right] \neq$ 0 cannot be isotopic to the identity on $A=A_{1} \cup A_{2}$, since the isotopy in $H^{1}(A, a)$ leaves $C_{1} \cup\{a\}$ pointwise fixed. Thus the isotopy classes of the collection of all such homeomorphisms $G_{1}^{-1} h$ on $A$ are $Z$. But since $G_{1}$ is $B$-isotopic to the identity fixing the point $a$, we have $\pi_{0}\left[H^{1}(A, a)\right] \cong$ $\pi_{0}\left[\left\{G_{1}^{-1} h\right\}\right]$ and the isotopy group is $Z$.

(iii) We note that, for every $h \in H^{2}(A, a)$, there is a homeomorphism $g$ such that $g \approx_{B} e$ on $A$ and $g^{-1} h=e$ on $\gamma \cup C_{1} \cup C_{2}$ by Lemma 2.5. The collection of the restricted homeomorphisms $\left\{g^{-1} h \mid A_{i}\right\}$, for all $h \in H^{2}(A, a)$ and the homeomorphisms $g$, generates the isotopy classes $Z$ for $i=1,2$ by arguments similar to that of the proof of (ii). Since the homeomorphisms $g$ are $B$-isotopic to the identity fixing the point $a$, we have $\pi_{0}\left[H^{2}(A, a)\right] \cong$ $\pi_{0}\left[\left\{g^{-1} h\right\}\right]$ and thus the isotopy group is $Z \times Z$.

In the following theorem, the solution for the case $n=0$ partially answers a question raised by Quintas [6, p. 932].

THEOREM 3.2. Let $D$ be $a$ disk and $a, x$ be two different points in $\operatorname{Int}(D)$. Then

$$
\begin{aligned}
\pi_{n}[H(D-a, x)] & =Z_{2} \quad \text { if } n=0, \\
& =0 \quad \text { if } n \geqq 1
\end{aligned}
$$

Proof. For the case $n=0$, for simplicity we consider $S^{1} \times(0,1]$ for $D-a$ and let $x=\left(0, \frac{1}{2}\right)$ and $C=S^{1} \times 1$. We first show that every $h \in H^{+}(D-a, x)$ is isotopic to the identity by an isotopy in $\mathrm{H}^{+}(D-a, x)$, where $\mathrm{H}^{+}(D-a, x)$ is the collection of the orientation preserving homeomorphisms in $H(D-a, x)$. Denote $\gamma=S^{1} \times \frac{1}{2}, B_{1}=S^{1} \times\left(0, \frac{1}{2}\right]$ and 
$B_{2}=S^{1} \times\left[\frac{1}{2}, 1\right]$. Then it can be seen that $h(\gamma)$ does not bound a disk or Möbius band and $h(\gamma) \simeq \gamma$ fixing the point $x$. Thus by Lemma 2.5 , there is an ambient isotopy $G_{t}: D-a, x \rightarrow D-a, x(0 \leqq t \leqq 1)$ such that $G_{0}=e$ and $G_{1}^{-1} h=e$ on the arc $\gamma$. Now observe that the restricted homeomorphism $G_{1}^{-1} h \mid B_{1}$ is isotopic to the identity on $B_{1}$. We can regard $G_{1}^{-1} h$ as a homeomorphism of a disk onto itself fixing one interior base point [5, Lemma 4.2]. Thus Lemma 2.1 implies that the homeomorphism $G_{1}^{-1} h$ is isotopic to the identity on the disk by an isotopy fixing the base point and the boundary $\gamma$ pointwise. In $B_{2}$, by Lemma 2.2 , it can be seen that the restricted homeomorphism $G_{1}^{-1} h \mid B_{2}$ is also isotopic to the identity on $B_{2}$ by an isotopy fixing the closed arc $\gamma$ pointwise and moving on the boundary $C$. Thus $G_{1}^{-1} h$ is isotopic to the identity on $D-a$ by an isotopy fixing the point $x$, and thus the homeomorphism $h$ is isotopic to the identity in $H^{+}(D-a, x)$. Hence we see that

$$
\pi_{0}[H(D-a, x)]=H(D-a, x) / H^{+}(D-a, x) \cong Z_{2}
$$

which completes the proof for the case $n=0$.

For the case $n \geqq 1$ we consider the homeotopy exact sequence. The sequence for $D-a$ yields $\pi_{n}[H(D-a)]=\pi_{n}[H(D-a, x)]$ for $n \geqq 2$ since $\pi_{n}(D-a, x)=0$ for $n \geqq 2$, and thus $\pi_{n}[H(D-a, x)]=0$ for $n \geqq 2[\mathbf{6}$, Theorem 5.1]. For $\pi_{1}[H(D-a, x)]$ we consider the end of the exact sequence,

$$
\begin{aligned}
\cdots & \rightarrow \pi_{2}(D-a, x) \rightarrow \pi_{1}[H(D-a, x)] \rightarrow \pi_{1}[H(D-a)] \\
& \rightarrow \pi_{1}(D-a, x) \rightarrow \pi_{0}[H(D-a, x)] \rightarrow \pi_{0}[H(D-a)] \rightarrow 0
\end{aligned}
$$

which is explicitly as follows.

$$
\cdots \longrightarrow 0 \stackrel{d_{*}}{\longrightarrow} \pi_{1}[H(D-a, x)] \stackrel{i_{*}}{\longrightarrow} Z \stackrel{P_{*}^{\prime}}{\longrightarrow} Z \stackrel{d_{*}}{\longrightarrow} Z_{2} \stackrel{i_{*}}{\longrightarrow} Z_{2} \stackrel{P_{*}}{\longrightarrow} 0 .
$$

In this sequence, since $P_{*}\left[\pi_{1}(H(D-a))\right]$ is the center of $\pi_{1}(D-a, x)=Z$, $P_{*}$ is an epimorphism which implies that it is in fact an isomorphism. Thus ker $P_{*}=0$ and $i_{*}\left[\pi_{1}(H(D-a, x))\right]=0$. Hence ker $i_{*}=\pi_{1}[H(D-a, x)]$, and since $d_{*}\left[\pi_{2}(D-a, x)\right]=0$, we obtain the result $\pi_{1}[H(D-a, x)]=0$. This completes the proof.

\section{REFERENCES}

1. J. W. Alexander, On the deformation of an n-cell, Proc. Nat. Acad. Sci. U.S.A. 9 (1923), 406-407.

2. D. B. A. Epstein, Curves on 2-manifolds and isotopies, Acta Math. 115 (1966), 83-107. MR 35 \#4938.

3. H. Gluck, The embedding of two-spheres in the four-sphere, Trans. Amer. Math. Soc. 104 (1962), 308-333. MR 26 \#4327. 
4. M. E. Hamstrom and E. Dyer, Regular mappings and the space of homeomorphisms on a 2-manifold, Duke Math. J. 25 (1958), 521-531. MR 20 \#2695.

5. G. S. McCarty, Jr., Homeotopy groups, Trans. Amer. Math. Soc. 106 (1962), 293-304. MR 26 \#3062.

6. L. V. Quintas, Solved and unsolved problems in the computation of homeotopy groups of 2-manifolds, Trans. New York Acad. Sci. 30 (1968), 919-938.

Department of Mathematics, Ohio State University, Lima, Ohio 45804

Current address: Department of Mathematics, State University of New York, Old Westbury, New York 11568 